\title{
Blood hyperviscosity with reduced skin blood flow in scleroderma
}

\author{
M. A. McGRATH, R. PEEK, AND R. PENNY
}

From the Department of Immunology, St. Vincent's Hospital, Sydney, and the Department of Medicine, University of New South Wales

SUMMARY The vascular complications of scleroderma have previously been attributed to the progressive obliteration of small vessels. Our study was carried out to determine whether abnormalities of blood viscosity occur in this disease, thereby contributing to the ischaemic process. Blood viscosity was measured in 20 patients using a rotational viscometer. At a high rate of shear, blood hyperviscosity was found in $35 \%$ of the patients and at a low rate of shear, in $70 \%$. In addition there was a significant increase in the plasma viscosity which implicates changes in plasma proteins (fibrinogen, immunoglobulins) as causing the hyperviscosity. Measurement of the hand blood flow by venous occlusion plethysmography showed reduced flow at $32^{\circ}, 27^{\circ}$, and $20^{\circ} \mathrm{C}$. A unique finding was a delayed recovery of the blood flow after cooling. These observations suggest that the increased resistance to blood flow in skin affected by scleroderma may be caused by an interaction between the occlusive vascular lesion and blood hyperviscosity. In addition, blood flow patterns and hyperviscosity could help distinguish scleroderma from primary Raynaud's disease.

In patients with scleroderma several of the clinical features, such as Raynaud's phenomenon, can be attributed to a reduced blood flow through small arteries, arterioles, and capillaries. Histological and radiological studies have confirmed the existence of vascular abnormalities in this disease (Soila, 1964; Norton and Nardo, 1970; Rodnan, 1972; Campbell and LeRoy; 1975), although the cause of these changes is unknown. Since the resistance to blood flow is partly dependent on the blood viscosity, detailed studies were carried out to determine whether blood hyperviscosity exists in patients with scleroderma, thereby contributing to the already increased vascular resistance associated with narrowing of the vessel lumen. The degree of impairment of the skin blood flow in this group of patients was assessed by measuring hand blood flow and its response to cooling. We compared the results with those obtained in patients with other connective tissue diseases and with impaired peripheral circulation.

\section{Patients}

Twenty patients with scleroderma were studied.

Accepted for publication April 28, 1977

Correspondence to Assoc. Prof. R. Penny, Dept. of Immunology, St. Vincent's Hospital, Darlinghurst 2010, Australia
Diagnosis was made clinically with support from appropriate laboratory and radiological investigations. These included skin histology, serum protein and autoantibody studies, barium studies of the upper gastrointestinal tract, and oesophageal motility measurements.

The severity of clinical involvement of the hand ranged from mild oedema with Raynaud's phenomenon to severe digital ischaemia. All vasoactive therapy was suspended at least 12 hours before the study. None of the patients were hypertensive or had undergone sympathectomy or intra-arterial therapy before the study.

\section{Methods}

BLOOD AND PLASMA VISCOSITY

Blood and plasma viscosities were measured using a rotational viscometer, the coaxial members of which have a rhombospheroid geometry (Dintenfass, 1969; McGrath, 1974). The speed of rotation-shear rate conversion factor was calculated from the geometry of the rhombospheroids as described elsewhere (Oka, 1960; McGrath, 1974). The instrument was calibrated using viscosity certified oils (Commonwealth Scientific and Industrial Research Organization, National Standards Laboratory, Sydney, Australia). Temperature control was 
obtained by means of a water jacket enclosing the rhombospheroids. All studies were carried out at $35^{\circ} \mathrm{C}$ and on blood anticoagulated with dry EDTA (1 $\mathrm{mg} / \mathrm{ml})$. The studies were completed within 3 hours of venepuncture. Blood viscosity was measured at 73 and $0.18 \mathrm{sec}^{-1}$, representing a high and low rate of shear respectively (Fig. 1), and the plasma viscosity was measured at 37 and $1.5 \mathrm{sec}^{-1}$. Technical limitations prevented the measurement of plasma viscosity at rates of shear above 37 and below $1.5 \mathrm{sec}^{-1}$.

The blood viscosity of 37 normal subjects was measured to determine the normal viscosity range for this instrument. In addition, the haematocrit was varied in 14 cases by the addition or removal of autologous plasma to permit a valid statistical analysis of the blood viscosity for haematocrits from 30 to $50 \%$. The linear regression line and tolerance limits for $\mathbf{P}=0.05$ were calculated on logarithmic

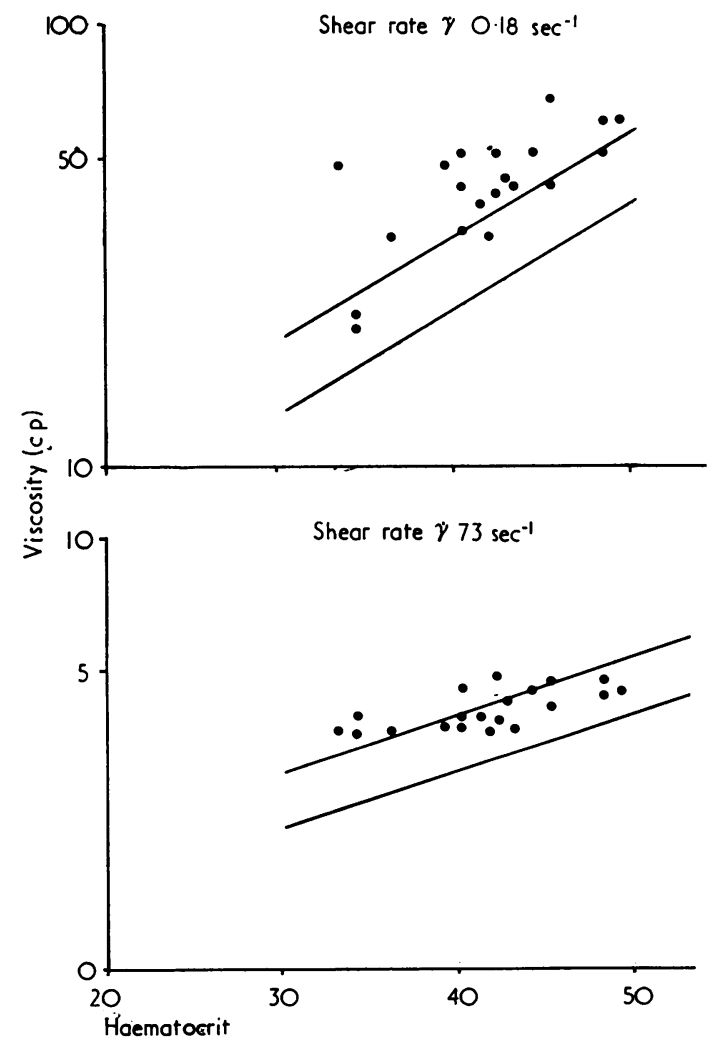

Fig. 1 Whole blood viscosity at low (top) and high (bottom) rates of shear in 20 patients with scleroderma. Tolerance limits $(P=0.05)$ for normal blood are indicated by the pairs of continuous lines. transformed viscosity data. Blood hyperviscosity defined as a blood viscosity value which is aboue these tolerance limits at the given haematocrit. The distribution of ages of the normal subjects was similar to the patients with scleroderma. The plasnia viscosity of 22 normal subjects was also measures.

Haematocrit was measured using heparinized microhaematocrit tubes (Sherwood). Premixed blood was drawn into the capillary tubes which were then centrifuged at $14000 \mathrm{~g}$ for 5 minutes (Clements Micro-Haematocrit centrifuge). The haematocrit was read to the nearest $0.5 \%$ using the Micro Haematg crit Capillary Tube Reader (Sherwood).

\section{HAND BLOOD FLOW}

Hand blood flows were measured by venous oeclusion plethysmography as described elsewhewe (Greenfield et al., 1963; Ardill et al., 1967). The changes in hand volume were recorded directly by the displacement of air above the water of the plethysmograph to a low-inertia float volume 18 corder and kymograph.

Before each experiment the patient lay supine fơr 30 minutes in a quiet, temperature-controlled room $\left(23^{\circ} \mathrm{C}\right)$ and all experiments were carried out ubder these conditions. During each experiment the temperature of the water in the plethysmograph enclosing the right hand was maintained at $32^{\circ} \mathrm{E}$ that the blood flows recorded from this hand served as a continuous control. This was necessary because, on occasions, the sequential hand blood flows varigd widely, apparently as the result of spontaneous changes in the peripheral sympathetic vascosstrictor tone. These fluctuations occurred synchronously in both hands and were approximately equ嘼] on both sides (Figs. 2, 3).

Blood flow measurements were recorded from the experimental (i.e. left) hand after sequential temperiture changes to $32^{\circ}, 27^{\circ}, 20^{\circ}$, and $32^{\circ} \mathrm{C}$. The recor ings were calibrated by the injection of known volumes of air into the tubing above the plethysmograph. The hand blood flows were calculated using the method described by Greenfield et al. (1963) and expressed as $\mathrm{ml}$ blood $/ 100 \mathrm{ml}$ hand tissue per minue. To arrive at an estimate of the net effect of each temperature change a correction procedure was applied which used the spontaneous fluctuations 兽 the control hand blood flows (Duff, 1952). to addition, the hand blood flows in 15 normal adit subjects (laboratory and hospital staff) were measured. Within this group there was no significant relationship between hand blood flow and age, thus supporting conclusions reached previousty (Shepherd, 1963).

Statistical analyses were performed using Studen 


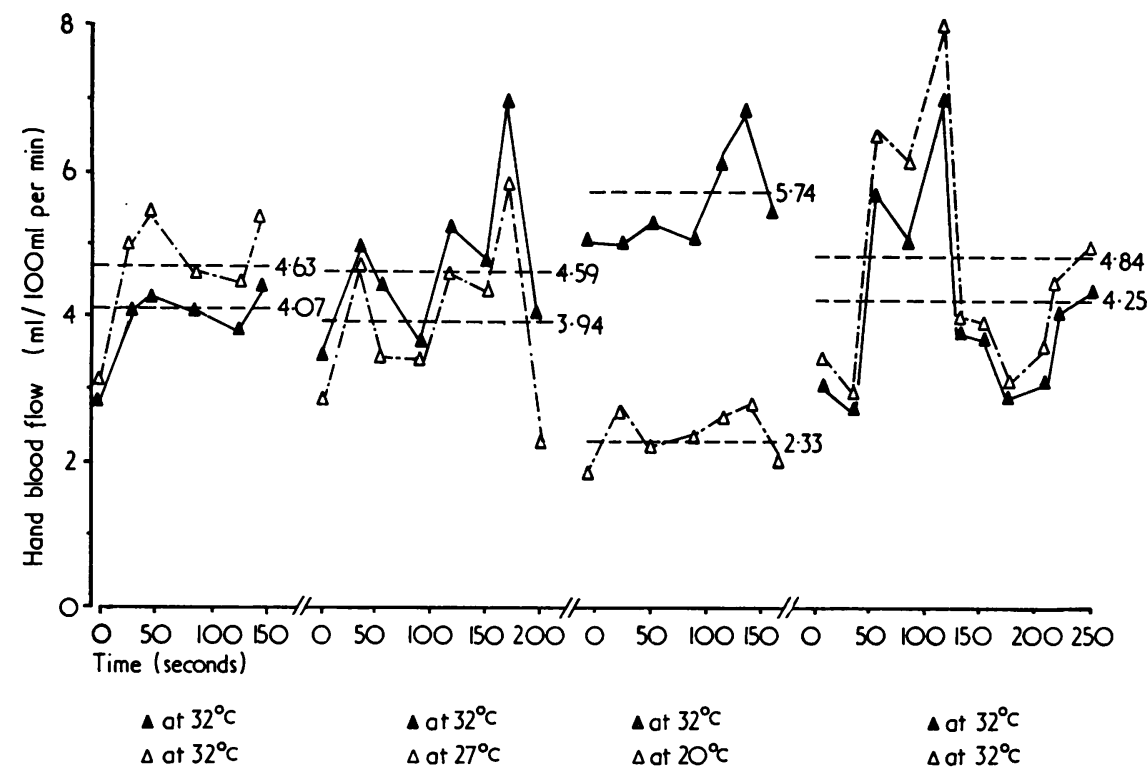

Fig. 2 Sequential hand blood flows in a normal subject, measured by venous occlusion plethysmography at different temperatures. The water in the plethysmograph enclosing the control (A right) hand was maintained at $32^{\circ} \mathrm{C}$ throughout the experiment. Recordings were started 4 minutes after each temperature change. $\Delta=$ left hand.

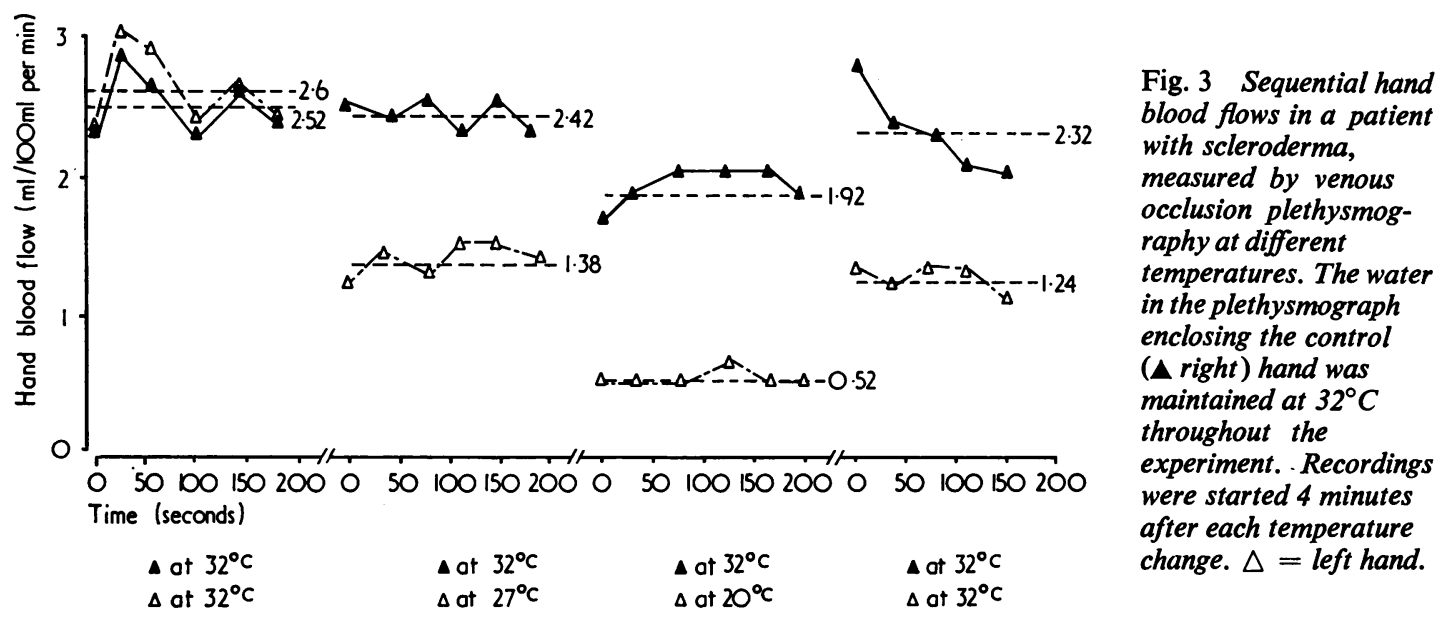

$t$ test. $\mathrm{P}<0.05$ was considered significant. As part of a comprehensive treatise (McGrath, 1974) hand blood flows and viscosity determinations were performed in 66 patients with paraproteinaemia, 21 patients with cryoglobulinaemia, 46 with polycythaemia, 18 with degenerative peripheral vascular disease, 22 with primary Raynaud's disease, 13 with systemic lupus erythematosus, 17 with cutaneous vasculitis, 38 with rheumatoid arthritis, and 79 with retinal vein occlusion. These results are presented elsewhere (McGrath and Penny, 1976; 1977a, b; McGrath et al., 1973) but referred to herein as considered relevant and to emphasize the significance and specificity of the observations in scleroderma.

\section{Results}

\section{BLOOD VISCOSITY}

Normal subjects

There was a linear correlation between the log viscosity and haematocrit for the range of haematocrits from 30 to $50 \%$. The tolerance limits for $\mathbf{P}=\mathbf{0 . 0 5}$ are shown in Fig. 1. Extrapolation of the regression line to zero haematocrit (i.e. plasma) gives a viscosity of 1.27 and 3.03 centipoise (cp) for 
73 and $0.18 \mathrm{sec}^{-1}$ respectively. These values agree with the normal plasma viscosity results obtained at $1.5 \mathrm{sec}^{-1}$ (see below). The relationship observed between viscosity and haematocrit is similar to that reported in other studies using different types of viscometer (Wells and Merrill, 1962; Rand et al., 1964; Chien et al., 1966; Dormandy, 1970; Copley, 1973).

\section{Scleroderma}

At a shear rate of $73 \mathrm{sec}^{-1} 7$ patients $(35 \%)$ had blood hyperviscosity and at $0.18 \mathrm{sec}^{-1}$ there were 14 patients $(70 \%)$ with hyperviscosity (Fig. 1).

\section{PLASMA VISCOSITY}

Normal subjects

Plasma viscosity at $37 \mathrm{sec}^{-1}$ was $1.39 \pm 0.10 \mathrm{cp}$ (mean $\pm \mathrm{SD}$ ) and at $1.5 \mathrm{sec}^{-1}$ was $2 \cdot 60 \pm 0.35 \mathrm{cp}$.

\section{Scleroderma}

Plasma viscosity in scleroderma was significantly increased $(P<0.005)$ above normal at both rates of shear, being $1.55 \pm 0 \cdot 15 \mathrm{cp}$ and $4.13 \pm 1.90 \mathrm{cp}$ at 37 and $1.5 \mathrm{sec}^{-1}$ respectively.

\section{HAND BLOOD FLOW}

Normal subjects

The hand blood flows at $32^{\circ}, 27^{\circ}$, and $20^{\circ} \mathrm{C}$ were $5.99 \pm 2.68$ (mean $\pm \mathrm{SD}$ ), $4.73 \pm 2.39$, and $2 \cdot 12$ $\pm 1.90 \mathrm{ml}$ blood $/ 100 \mathrm{ml}$ tissue per min respectively. Cooling of the hand from $32^{\circ}$ to $27^{\circ} \mathrm{C}$ resulted in a $27 \cdot 2 \pm 4.6 \%$ (mean $\pm \mathrm{SE}$ ) decrease in flow, and cooling from $32^{\circ}$ to $20^{\circ}$ resulted in a $63.8 \pm 4.6 \%$ decrease. Therefore the change in hand blood flow over this temperature range is approximately $5 \%$ per centigrade degree. After rewarming of the hand from $20^{\circ}$ to $32^{\circ} \mathrm{C}$ the hand blood flow recovered to within $1 \cdot 1 \pm 2 \cdot 2 \%$ of control (Figs. 2, 4).

\section{Scleroderma}

The hand blood flows at $32^{\circ}, 27^{\circ}$, and $20^{\circ} \mathrm{C}$ were $3 \cdot 29 \pm 2 \cdot 33$ (mean \pm SD), $2 \cdot 20 \pm 1 \cdot 76$, and $1 \cdot 00 \pm$ $0.75 \mathrm{ml}$ blood $/ 100 \mathrm{ml}$ tissue per min respectively. These values are all significantly below $(\mathrm{P}<0.005)$ the corresponding values in the normal subjects. The decreases in blood flow with cooling from $32^{\circ}$ to $27^{\circ}$ and from $32^{\circ}$ to $20^{\circ} \mathrm{C}$ were $31 \cdot 5 \pm 2 \cdot 7 \%$ (mean $\pm \mathrm{SE}$ ) and $66 \cdot 3 \pm 3 \cdot 2 \%$ respectively. These values are not significantly different from the corresponding results obtained in the normal group. By contrast, the degree of recovery of the hand blood flow in scleroderma with rewarming from $20^{\circ}$ to $32^{\circ} \mathrm{C}$ was only 60.5 $\pm 4.6 \%$, which is significantly below $(P<0.001)$ the recovery in the normal group (Fig. 3, 4). For comparison, the results of blood flow changes are shown

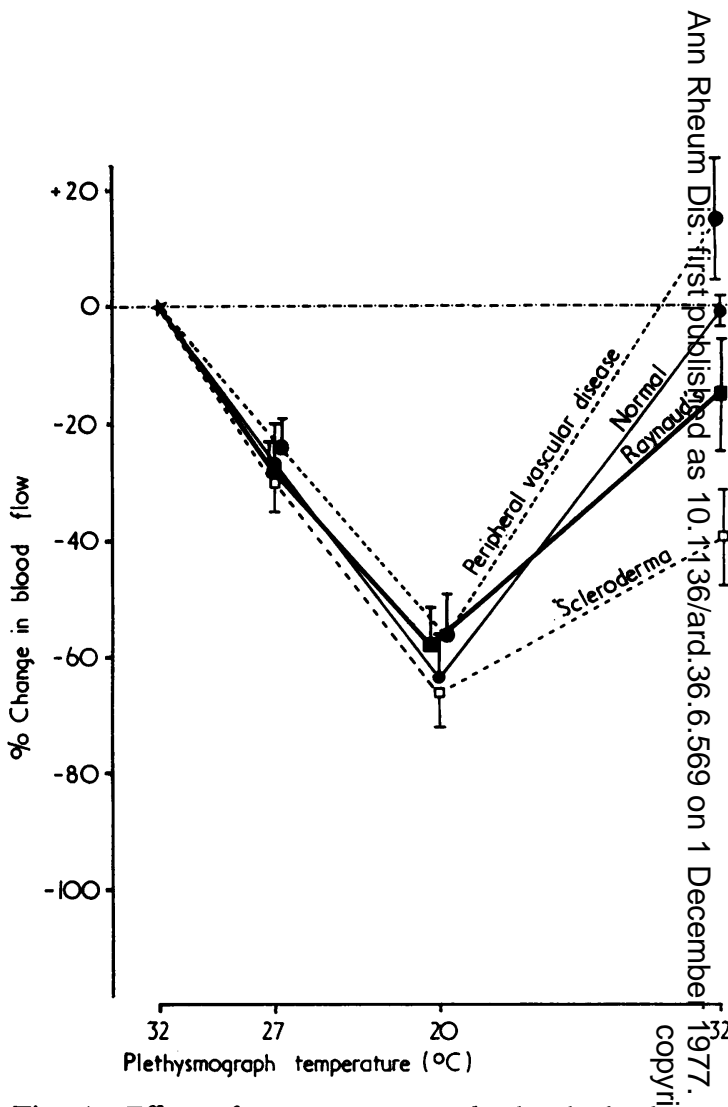

Fig. 4 Effect of temperature on the hand bloodofigh in normal subjects $(n=15)$ and in patients with sete derma $(n=20)$. The per cent change in blood flow (metn $\pm S E$ ) was corrected for spontaneous fluctuations (see texo). The difference between the two groups after rewarm (from $20^{\circ} \mathrm{C}$ to $32^{\circ} \mathrm{C}$ ) is significant $(P<0.001)$. Fr comparison, results are included for patients with periphe $\overline{\vec{a}}$ l vascular disease $(n=18)$ and those with primary Răynaud's disease $(n=22)$. Differences from scleroder patients after rewarming are significant.

for patients with peripheral vascular disease amd primary Raynaud's disease. Of all groups of diseases studied, scleroderma alone produced such a pattep̧n of response.

\section{Discussion}

By means of a rotational viscometer it is possible measure the viscosity of whole blood at a uniform rate of shear and hence determine accurately the intrinsic resistance to flow offered by a particulat sample of blood under given conditions of flow. The instrument used in our study has the capacity 40 measure the blood viscosity at both high and low rates of shear and therefore the contributions of haematocrit and red cell aggregation to the blogd viscosity can be determined separately. Interpretition of the results is supported by measurements of 
the plasma viscosity and haematocrit; an increase in the former indicates a change in the plasma proteins and since certain plasma proteins promote red cell aggregation, is more likely to be associated with blood hyperviscosity at low rates of shear; whereas an increase in the haematocrit predisposes to blood hyperviscosity at both high and low rates of shear (Chien et al., 1966).

The validity of the measurements obtained with the instrument used in the present study has previously been established by comparison of the results obtained in a normal group of subjects with the normal viscosity ranges reported by other investigators using different viscometers (McGrath, 1974). In addition, the presentation of data in the manner illustrated allows a ready evaluation of the results relative to the normal range for each given haematocrit. The temperature of each sample was carefully controlled during the recordings since the viscosity of fluids increases with decreasing temperature.

The high incidence of blood hyperviscosity found in these patients suggests that this abnormality may contribute significantly to the vascular complications of scleroderma. In particular the observation that the majority of patients had blood hyperviscosity at low rates of shear draws attention to the high resistance offered by the blood, especially at low flow rates. The significant increase in the plasma viscosity, which implies that there is either an increase or a physicochemical change in the macromolecular components of plasma, is consistent with the greater incidence of blood hyperviscosity at low rates of shear.

Whether blood hyperviscosity in scleroderma is the result of a primary haematological abnormality or is secondary to the disease process cannot be determined on the available evidence. Increased red cell aggregation has been reported in association with a variety of forms of tissue injury (Davis and Landau, 1966; Charm and Kurland, 1972; Chien, 1975), but the mechanism of the aggregation remains speculative. In some cases it is apparently related to fibrinogen, in some to other plasma proteins, and in others it has been attributed to altered properties of the red cell surface.

Although these factors were not analysed in the present study other investigations have confirmed that serological abnormalities are frequent in scleroderma and include hypergammaglobulinaemia (Clark et al., 1971; Rodnan, 1972) and rheumatoid factor (Clark et al., 1971; Rodnan, 1972). Such changes are consistent with the raised erythrocyte sedimentation rate (Clark et al., 1971) and increased intravascular red cell aggregation (Davis and Landau, 1966; Redisch et al., 1970) in this disease.
In our patients, raised ESR, fibrinogen, immunoglobulins, and cryoproteins were uniformly associated with hyperviscosity. At least in paraproteinaemia a very close relationship existed between hyperviscosity and immunoglobulin concentration (McGrath and Penny, 1976). However, no clear correlation existed between disease activity, disease extent, antinuclear antibodies, rheumatoid factor, and hyperviscosity in scleroderma. In fact, our data have perhaps more relevance to hyperviscosity being an added mechanism of ischaemia rather than being a laboratory aid in diagnosis, except in distinguishing Raynaud's disease.

Hyperviscosity is present in many patients with connective tissue diseases with comparable prevalence and therefore scleroderma is not separable on these grounds. Yet it does highlight an infrequently appreciated phenomenon in all these disorders (McGrath, 1974). However, none of the patients fulfilling the criteria for a diagnosis of primary Raynaud's disease had hyperviscosity (McGrath et al., 1977b). Measurement of viscosity therefore would be of considerable help in this latter clinical situation to distinguish early scleroderma.

The abnormal blood vessels in scleroderma skin predisposes to slow blood flows and low rates of shear. Since the blood viscosity increases disproportionally with decreasing rate of shear there will be regions of the skin circulation in which the viscous resistance is abnormally high. Under these conditions local haemodynamic forces may be of insufficient magnitude to maintain flow resulting in stasis, vascular occlusion, and tissue ischaemia. Although such a sequence remains speculative it is consistent with the results of the hand blood flow measurements and, in particular, with the abnormally slow recovery of the skin blood flow after exposure to cold. The hand blood flow observations presented in Fig. 4 are completely unique to scleroderma. None of the disease groups analysed showed such a defective recovery. Therefore, scleroderma could be distinguished from SLE, rheumatoid arthritis, and primary Raynaud's disease, all of which have normal flow recoveries after cold exposure (McGrath, 1974; McGrath et al., 1977b).

The reduced hand flow rates reflect severe increases in the resistance to flow and confirm previous observations that there are marked - abnormalities of the peripheral circulation in this disease. The mechanism of the slow recovery after cold could be a prolonged cold-induced vasoconstriction or a failure of the intravascular cellular aggregates formed during the cold to disperse, or to a combination of these phenomena. Although the 
precise mechanism cannot be identified from the present study the association of blood hyperviscosity suggests a causative role, at lease in part, for intravascular red cell aggregation.

These observations therefore support a multifactorial basis for the frequent vascular complications of scleroderma since the coexistence of blood vessel abnormalities and blood hyperviscosity will result in a higher resistance to blood flow than either factor alone. In addition, we suggest that this combination is responsible for the progressive nature of the vascular changes in this disease.

M. A. McG. was supported by National Health and Medical Research Council of Australia Medical Postgraduate Research Fellowship.

\section{References}

Ardill, B. L., Bhatnagar, U. M., Fentem, P. H., and Greenfield, A. D. M. (1967). Clinical use of venous occlusion plethysmography. Scandanavian Journal of Clinical and Laboratory Investigation, 99, Suppl., 95-100.

Campbell, P. M., and LeRoy, E. C. (1975). Pathogenesis of systemic sclerosis: a vascular hypothesis. Seminars in Arthritis and Rheumatism, 4, 351-368.

Charm, S. E., and Kurland, G. S. (1972). Blood rheology. Cardiovascular Fluid Dynamics, Vol. 2, p. 157. Ed. by D. H. Bergel. Academic Press, New York.

Chien, S. (1975). Biophysical behaviour of red cells in suspensions. The Red Blood Cell, 2nd ed., p. 1031. Ed. by D. MacN. Surgenor. Academic Press, New York.

Chien, S., Usami, S., Taylor, H. M., Lundberg, J. L., and Gregersen, M. I. (1966). Effects of hematocrit and plasma proteins on human blood rheology at low shear rates. Journal of Applied Physiology, 21, 81-87.

Clark, J. A., Winkelmann, R. K., and Ward, L. E. (1971). Serologic alterations in scleroderma and sclerodermatomyositis. Mayo Clinic Proceedings, 46, 104-107.

Copley, A. L. (1973). On biorheology. Biorheology, 10, 87-105.

Davis, E., and Landau, J. (1966). Journal of Clinical Capillary Microscopy, p. 53 and p. 149. Thomas, Springfield, Illinois.

Dintenfass, L. (1969). A coaxial rhombospheroid viscometer: a further development of the cone-in-cone viscometer. Biorheolcgy, 6, 33-36.

Dormandy, J. (1970). Clinical significance of blood viscosity. Annals of the Royal College of Surgeons of England, 47, 211-228.

Duff, R. S. (1952). Effect of sympathectomy on the response to adrenaline of the blood vessels of the skin in man. Journal of Physiology, 117, 415-430.
Greenfield, A. D. M., Whitney, R. J., and Mowbray, J. (1963). Methods for the investigation of peripheral bloof flow. British Medical Bulletin, 19, 101-109.

McGrath, M. A. (1974). A correlative study of haemor heological, immunological and clinical parameters in disorders of blood flow. MD thesis, University of Net South Wales, Australia.

McGrath, M. A., and Penny, R. (1976). Paraproteinemia blood hyperviscosity and clinical manifestations. Journat of Clinical Investigation, 58, 1155-1162.

McGrath, M. A., and Penny, R. (1977a). Blood hyperviscositỹ in cryoglobulinaemia: temperature sensitivity and correlation with reduced skin blood flow. Australian Journal of Experimental Biology and Medical Science (in press).

McGrath, M. A., and Penny, R. (1977b). The relationship between blood viscosity and haematocrit in normad subjects and in polycythaemia. (Submitted for publicao

tion).
McGrath, M. A., Tracy, G. D., Lord, R. S. A., and Penny, Ro (1973). Peripheral ischaemia caused by blood hyperviscosity. Australian and New Zealand Journal of Surgeryo 43, 109-113.

McGrath, M. A., Wechsler, F., Hunyor, A. B. L., and Penny, R. (1977a). Systemic factors contributory to retinal vein occlusion. Archives of Internal Medicine (in press).

McGrath, M. A., Peek, R., and Penny, R. (1977b). Raynaud disease: reduced hand blood flows with normal viscosit

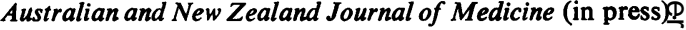

Norton, W. L., and Nardo, J. M. (1970). Vascular disease in progressive systemic sclerosis (scleroderma). Annal: Internal Medicine, 73, 317-324.

Oka, S. (1960). The principles of rheometry. Rheolög Vol. 3, p. 18. Ed. by F. R. Eirich. Academic Press, York.

Rand, P. W., Lacombe, E., Hunt, H. E., and Austir W. H. (1964). Viscosity of normal human blood undee normothermic and hypothermic conditions. Journal \& Applied Physiology, 19, 117-122.

Redisch, W., Messina, E. J., Hughes, G., and McEwan, (1970). Capillaroscopic observations in rheumatic diseases Annals of the Rheumatic Diseases, 29, 244-252.

Rodnan, G. P. (1972). Progressive systemic sclerosip. Arthritis and Allied Conditions, 8th ed., p. 962. Ed. bo J. L. Hollander and D. J. McCarthy. Lea and Febige Philadelphia.

Shepherd, J. T. (1963). Physiology of the Circulation Human Limbs in Health and Disease, p. 201. Saundersi Philadelphia.

Soila. P. (1964). Some features of angiographic findings in rheumatoid arthritis and scleroderma. Acta Rheumatos logica Scandinavica, 10, 189.

Wells, R. E., and Merrill, E. W. (1962). Influence of flo properties of blood upon viscosity - haematocrit relationships. Journal of Clinical Investigation, 41, 1591-1598. O 